\title{
Optoelectronic Logic Functions Based on Reconfigurable SiC Multilayer Devices
}

\author{
Manuel A. Vieira ${ }^{1,2}$, Vitor Silva ${ }^{1,2}$, Paula Louro ${ }^{1,2}$, Manuela Vieira ${ }^{1,2,3}$, \\ and Manuel Barata ${ }^{1,2}$ \\ ${ }^{1}$ Electronics Telecommunications and Computer Dept, ISEL, Lisbon, Portugal \\ ${ }^{2}$ CTS-UNINOVA, Quinta da Torre, 2829-516, Caparica, Portugal \\ ${ }^{3}$ DEE-FCT-UNL, Quinta da Torre, 2829-516, Caparica, Portugal
}

\begin{abstract}
WDM multilayered $\mathrm{SiC} / \mathrm{Si}$ devices based on a-Si:H and a-SiC:H filter design are approached from a reconfigurable point of view. Results show that the devices, under appropriated optical bias, act as reconfigurable active filters that allow optical switching and optoelectronic logic functions development. Under front violet irradiation the magnitude of the red and green channels are amplified and the blue and violet reduced. Violet back irradiation cuts the red channel, slightly influences the magnitude of the green and blue ones and strongly amplifies de violet channel. This nonlinearity provides the possibility for selective removal of useless wavelengths. Particular attention is given to the amplification coefficient weights, which allow taking into account the wavelength background effects when a band needs to be filtered from a wider range of mixed signals, or when optical active filter gates are used to select and filter input signals to specific output ports in WDM communication systems. A truth table of an encoder that performs 8-to-1 multiplexer (MUX) function is presented.
\end{abstract}

Keywords: Optoelectronic logic functions, Reconfigurable devices, Numerical simulation, Transfer characteristics, Coder-encoder devices, Encoder truth tables.

\section{Introduction}

Optical communication in the visible spectrum usually interfaces with an optoelectronic device for further signal processing. There has been much research on semiconductor devices as elements for optical communication [1, 2, 3].

Multilayered structures based on amorphous silicon technology are predictable to become reconfigurable to perform WDM optoelectronic logic functions and provide photonic functions such as signal amplification and switching [4]. They will be a solution in WDM technique for information transmission and decoding in the visible range [5]. Amplification and amplitude change are two key functionality properties outcome of a balanced interaction between wavelength of the optical signal and background wavelength and placement within a WDM link. Any change in any of these factors will result in filter readjustments. An algorithm to decode the information is presented and support new optoelectronic logic architecture. 


\section{Internet of Things}

Future advances in computer speeds are contingent on augmenting intra- and interchip bandwidth. Increases in power efficiency per bit of data is projected to be achieved by replacing electrical interconnects with their optical counterparts in the near future. The implementation of photonic circuits for the purpose of chip-level communications requires devices such as modulators, detectors and wavelength division multiplexers (WDM).

Reconfigurable multi-rate next generation optical networks are currently investigated to handle the ever increasing growth of the Internet traffic. Reconfigurable wavelength selectors that allow for operation on a large number of wavelength channels, with dynamic response, are essential sub-systems for implementing reconfigurable WDM networks and optical signal processing.

In this paper, we demonstrate that is possible, using the amorphous $\mathrm{Si} / \mathrm{C}$ technology, to produce reconfigurable $\mathrm{SiC}$ multilayer devices acting simultaneously as photodetectors, selectors and active filters. Therefore, the theoretical approach presented in this research is useful in examining a particular aspect of the optical communications, configuring firm activities towards innovation and combining unique resources and capabilities. Devices based on new materials for signal (de)multiplexing in the visible spectrum are an important contribute to the future development of the Internet of Things.

\section{Device Configuration and Operation}

The device consists of a p-i'(a-SiC:H)-n/p-i(a-Si:H)-n heterostructure with low conductivity doped layers (Fig.1). The thicknesses and optical gap of the front 1'- (200 $\mathrm{nm} ; 2.1 \mathrm{eV})$ and back i- (1000 nm; $1.8 \mathrm{eV})$ layers are optimized for light absorption in the blue and red ranges, respectively $[6,7]$. As a result, both front and back pin structures act as optical filters confining, respectively, the blue and the red optical carriers.

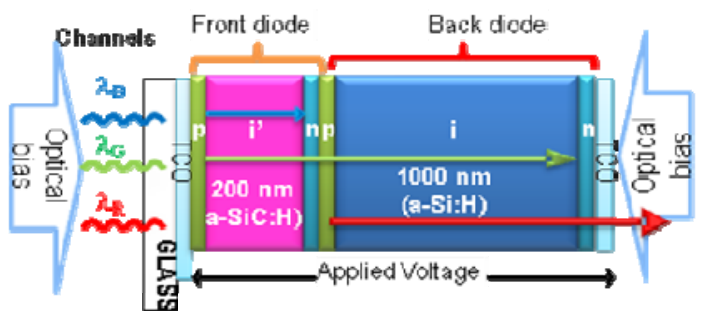

Fig. 1. Device configuration and operation

Several monochromatic pulsed lights, separately $\left(\lambda_{\mathrm{R}, \mathrm{G}, \mathrm{B}}\right.$ input channels) or in a polychromatic mixture (multiplexed signal) at different bit rates illuminated the device from the glass side. Steady state optical bias with different wavelength are superimposed $(400 \mathrm{~nm}-800 \mathrm{~nm})$ from the front or from the back sides and the generated photocurrent measured at $-8 \mathrm{~V}$. The device operates within the visible range 
using as input color channels (data) the wave square modulated light (external regulation of frequency and intensity) supplied by a red (R: $\left.626 \mathrm{~nm} ; 51 \mu \mathrm{W} / \mathrm{cm}^{2}\right)$, a green (G: $524 \mathrm{~nm} ; 73 \mu \mathrm{W} / \mathrm{cm}^{2}$ ) and a blue (B: $470 \mathrm{~nm} ; 115 \mu \mathrm{W} / \mathrm{cm}^{2}$ ) LED. Additionally, steady state red, green, blue and violet (background) was superimposed by different LED's (R: $625 \mu \mathrm{W} / \mathrm{cm}^{2}$, G: $515 \mu \mathrm{W} / \mathrm{cm}^{2}, \mathrm{~B}: 400 \mu \mathrm{W} / \mathrm{cm}^{2}, \mathrm{~V}: 2800$ $\left.\mu \mathrm{W} / \mathrm{cm}^{2}\right)$.

\section{$4 \quad$ Photonic Active Filters}

The spectral sensitivity was tested through spectral response measurements under different frequencies, with and without steady state optical bias applied either from the front or back side.
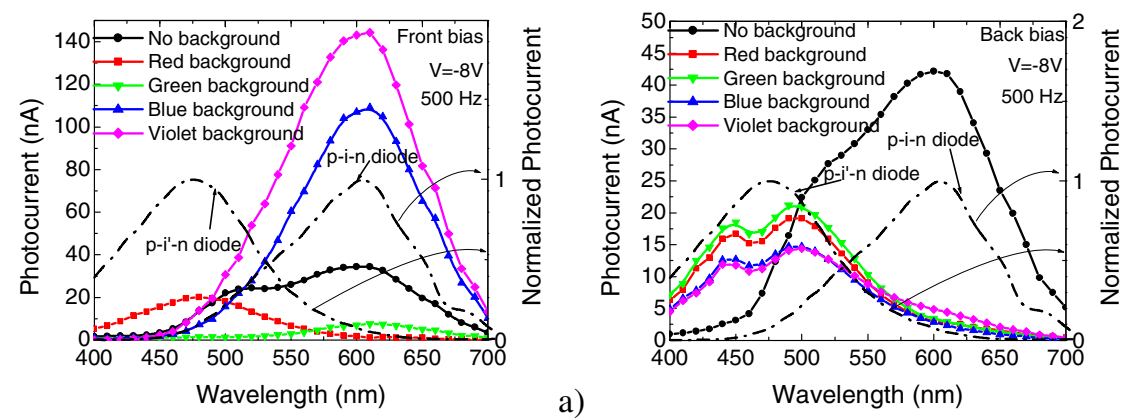

b)

Fig. 2. Photocurrent without and with front (a) and back (b) backgrounds. The current of the individual photodiodes are superimposed (dash lines).

In Fig. 2, the spectral photocurrent (symbols) is displayed under red, green, blue and violet background and without it. In Fig. 2a the steady state optical bias was applied from the front side and in Fig. $2 b$ from the back side. For comparison the normalized spectral photocurrent for the front, $\mathrm{p}-\mathrm{i}$ '- $\mathrm{n}$, and the back, $\mathrm{p}-\mathrm{i}-\mathrm{n}$, photodiodes (dash lines) are superimposed.

Data shows that the front and back diodes, separately, presents the typical response of single p-i-n cells with intrinsic layers based on a-SiC:H or a-Si:H materials, respectively. The front diode cuts the wavelengths higher than $550 \mathrm{~nm}$ while the back one rejects the ones lower than $500 \mathrm{~nm}$. The overall device presents an enlarged sensitivity when compared with the individual ones.

Under front irradiation the sensitivity is much higher than under back irradiation. Under front irradiation (Fig. 2a) the violet background amplifies the spectral sensitivity in the visible range while the blue optical bias only enhances the spectral sensitivity in the long wavelength range $(>550 \mathrm{~nm})$ and quenches it in the others. Under red bias, the photocurrent is strongly enhanced at short wavelengths and disappears for wavelengths higher than $550 \mathrm{~nm}$. Under green the sensitivity is strongly reduced in all the visible spectra. In Fig. 2b, whatever the wavelength of the 
backgrounds, the back irradiation strongly quenches the sensitivity in the long wavelength range $(>550 \mathrm{~nm})$ and enhances the short wavelength range. So, back irradiation, tunes the front diode while front irradiation, depending on the wavelength used, can tune the back one.

In Fig. 3 the spectral gain, defined as the ratio between the spectral photocurrents under red $\left(\alpha^{\mathrm{R}}\right)$, green $\left(\alpha^{\mathrm{G}}\right)$ blue $\left(\alpha^{\mathrm{B}}\right)$ and violet $\left(\alpha^{\mathrm{V}}\right)$ illumination and without it is plotted at $3500 \mathrm{~Hz}$. The optical bias is applied from the front side, in Fig. 3a and from the back side, in Fig. 3b.
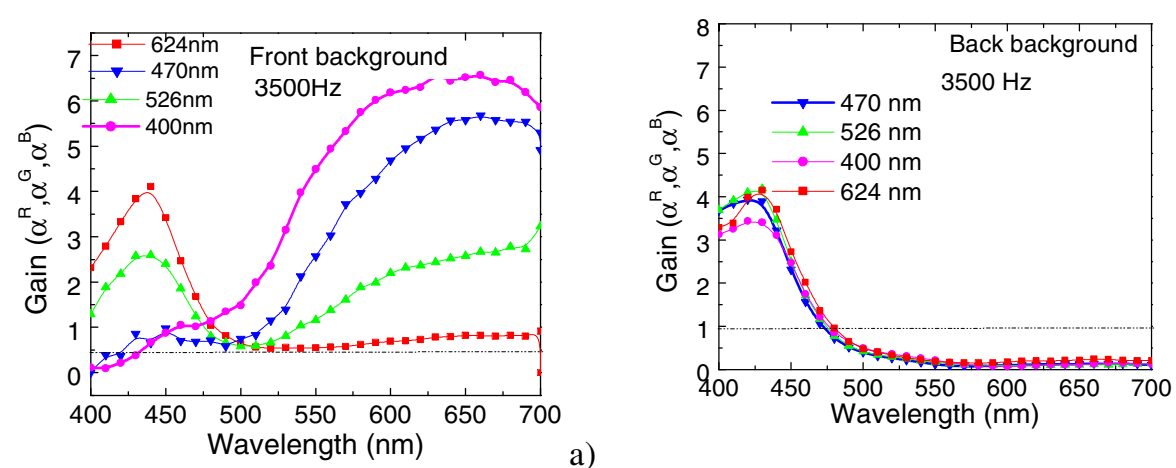

a)

Fig. 3. Spectral gain under red $\left(\alpha^{\mathrm{R}}\right)$, green $\left(\alpha^{\mathrm{G}}\right)$, and blue $\left(\alpha^{\mathrm{B}}\right)$ optical bias, applied from the front (a) and the back (b) sides at $3500 \mathrm{~Hz}$

Under front bias and red irradiation, the gain is high at short wavelengths and strongly lowers for wavelengths higher than $500 \mathrm{~nm}$, acting as a short-pass filter. Under green background, the device behaves as a band-stop filter that screens out the medium wavelength range $(475 \mathrm{~nm}-550 \mathrm{~nm})$ enhancing only the photocurrent for wavelengths outside of that range. Under blue and violet light the device works as a long-pass filter for wavelengths higher than $550 \mathrm{~nm}$, blocking the shorter wavelengths. Back light, whatever the frequency, leads to a short-pass filter performance. Results show that by combining the background wavelengths and the irradiation side, the short-, medium- and longspectral region can be sequentially tuned.

\section{$5 \quad$ Encoder and Decoder Device}

To analyze the device under information-modulated wave and uniform irradiation, three monochromatic pulsed lights separately (red, green and blue input channels, Fig. 4) or combined (multiplexed signal, Fig.5) illuminated the device at 6000 bps. Steady state violet optical bias was superimposed separately from the front (solid lines) and the back (dash lines) sides and the photocurrent generated measured at $-8 \mathrm{~V}$. The transient signals were normalized to their values without background. 

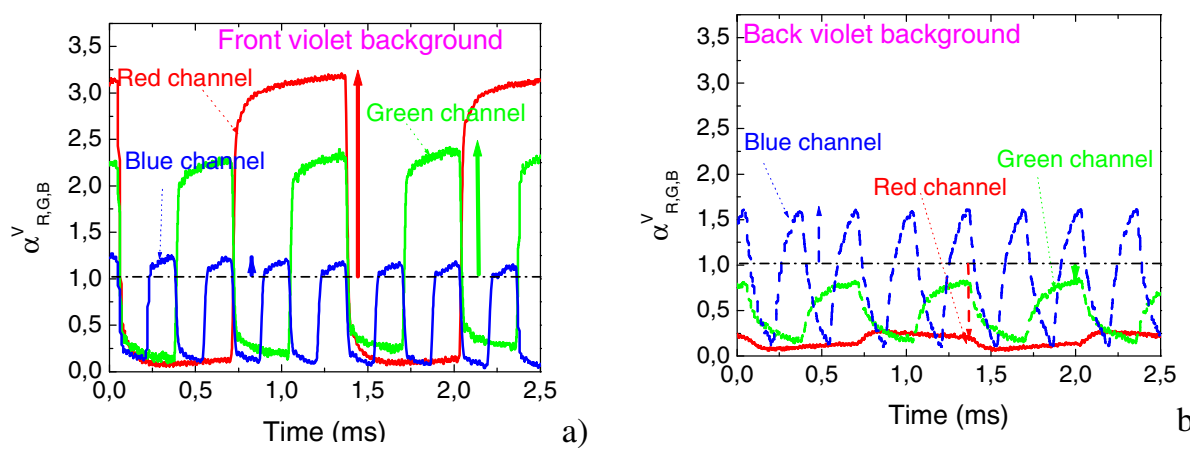

Fig. 4. Normalized red, green and blue transient signals at $-8 \mathrm{~V}$ with violet (400 nm) steady state optical bias applied from the front and the back sides

Results show that, even under transient conditions, the background side affects the signal magnitude of the color channels. Under front irradiation, it enhances mainly the light-to-dark sensitivity in the medium-long wavelength ranges. Violet radiation is absorbed at the top of the front diode, increasing the electric field at the least absorbing cell, the back diode. Taking into account Fig. 4, the red and green collections are strongly enhanced $\left(\alpha_{\mathrm{G}}^{\mathrm{V}}=2.2, \alpha_{\mathrm{R}}^{\mathrm{V}}=3.1\right)$ while the blue one stays near its dark value $\left(\alpha_{B}^{\mathrm{V}}=1.1\right)$. Under back irradiation the small absorption depth of the violet photons across the back diode quenches the electric field and so, the red collection almost disappears $\left(\alpha_{\mathrm{R}}^{\mathrm{V}}=0.2\right)$. Here, blue channel is absorbed across the front diode where the electric field is enhanced resulting in an increase collection of the blue channel $\left(\alpha_{B}^{\mathrm{V}}=1.6\right)$. Since the green channel is absorbed across front and back diodes its collection is balanced by the increased collection in the front diode and its reduction at the back one $\left(\alpha_{\mathrm{G}}^{\mathrm{V}}=0.7\right)$.

For an optoelectronic digital capture system, opto-electronic conversion is the relationship between the optical inputs and the corresponding digital output levels. In Fig. 5 the normalized multiplexed signal under front (symbols) and back (lines) violet irradiation is displayed. On the top of the figure the signals used to drive the input channels are shown to guide the eyes into the ON/OFF channel states.

Under front irradiation ( $\operatorname{pin}_{1} ; \alpha_{\mathrm{R}}>>1, \alpha_{\mathrm{G}}>1$ and $\alpha^{\mathrm{V}}{ }_{\mathrm{B}} \sim 1$ ) the $2^{3}$ levels can be grouped into two main classes due to the high amplification of the red channel. The upper four levels are ascribed to the presence of the red channel ON, and the lower four to its absence, allowing the red channel decoder (4-to-1 multiplexer; long-pass filter function). Since under front irradiation the green channel is amplified the two highest levels, in both classes, are ascribed to the presence of the green channel and the two lower ones to its lack.

Under back irradiation ( $\operatorname{pin}_{2} ; \alpha^{\mathrm{V}}{ }_{\mathrm{R}}<<1, \alpha^{\mathrm{V}}{ }_{\mathrm{G}}<1$ and $\alpha^{\mathrm{V}}{ }_{\mathrm{B}}>1$, Fig. 4), the blue channel is enhanced; the green reduced the red almost suppressed. The encoded multiplexed signal has the eight sublevels grouped also into two main levels, the higher where the blue channel is ON and the lower where it is OFF (4-to-1 multiplexer; short-pass filter 


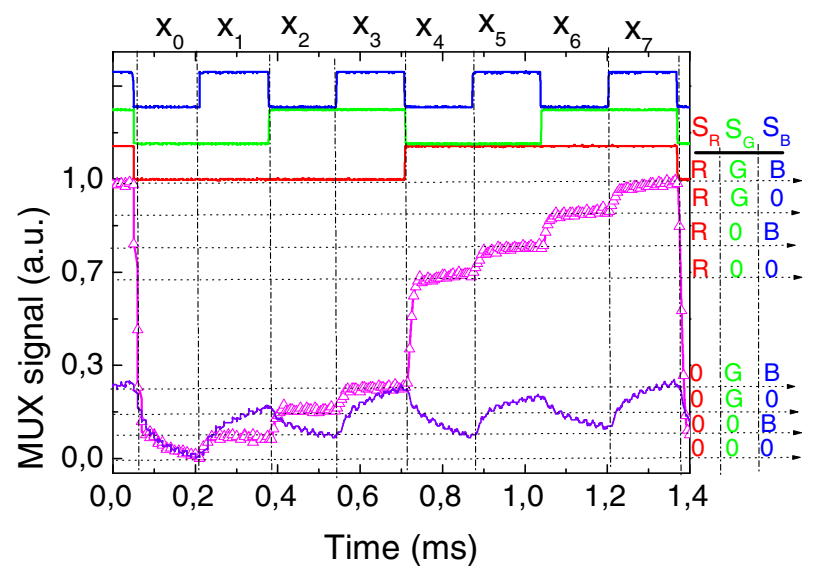

Fig. 5. Normalized multiplexed signal under front (symbols) and back (lines) violet irradiation. On the top the signals used to drive the input channels are shown to guide the eyes into the ON/OFF channel states.

function). In each main level the four existent sublevels are grouped in two classes, with and without the channel green ON. Each of those sublevels split into two near ones, attributed to the presence or absence of the red channel. If we consider this red output bit "not significant" only four separate levels $\left(2^{2}\right)$ are considered and the logic MUX function is converted into a logic filter function. The blue channel is then decoded. This decoding algorithm is mapped in the right side of the multiplexed signal using a RGB code for each level (Fig. 5).

Like regular binary numbers, the binary RGB code is an arithmetic code and so, it is weighted, $i$. $e$. there is specific weights assigned to each bit position. Under front violet irradiation, the most significant digit, the left most bit, in the RGB code, is the $\operatorname{red}\left(\alpha_{\mathrm{R}}^{\mathrm{V}}>>1\right)$. Going from the left to right, the next is the green $\left(\alpha_{\mathrm{G}}^{\mathrm{V}}>1\right)$ and the last is the blue $\left(\alpha_{\text {Bpin } 1}^{\mathrm{V}} 1\right)$. Under back violet irradiation, the left most bit is the blue, the next is the green and last the red.

The truth tables of both encoders of Fig. 6, that perform 8-to-1 MUX function, are shown in Fig. 6. In the inputs $\left(\mathrm{x}_{0} \ldots \mathrm{x}_{7}\right)$, the index of each bit, is related to the first (highest) nonzero logic input. Here, the MUX device selects, through the violet background, one of the eight possible input logic signals and sends it to the output $\left(\mathrm{y}=\mathrm{x}_{\mathrm{S}}\right)$. The output is a three-bit $\left[\mathrm{S}_{2} \mathrm{~S}_{1} \mathrm{~S}_{0}\right]$ binary RGB number that may identify one of eight possible inputs.

Results show that the correspondence between the on/off state of the input channels $S_{R}, S_{G}, S_{B}$ in Fig. 5 and $S_{2}, S_{1}, S_{0}$, outputs in Fig. 6, are obvious. In Fig. 6 , for the input $x_{7}$ and output $S_{2}$, the first nonzero logic input is $7\left(2^{2}+2^{1}+2^{0}\right)$, which corresponds an output [111]. Those OR gates are expressed, respectively, as: $\mathrm{S}_{2}=\mathrm{x}_{7}+\mathrm{x}_{6}+\mathrm{x}_{5}+\mathrm{x}_{4}$ under front irradiation (Fig. 6a) and $\mathrm{S}_{2}=\mathrm{x}_{7}+\mathrm{x}_{3}+\mathrm{x}_{5}+\mathrm{x}_{1}$ under back light (Fig. 6b). 


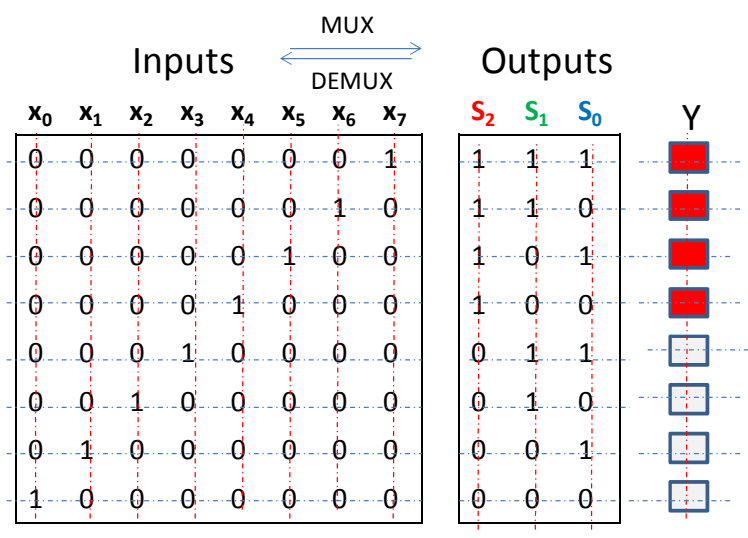

a)

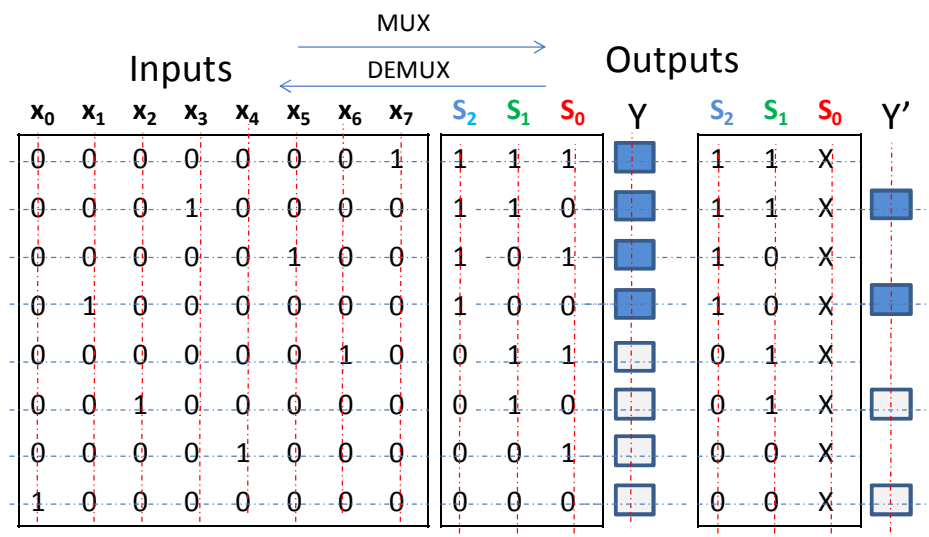

b)

Fig. 6. Truth tables of the encoders that perform 8-to-1 multiplexer (MUX) function, under (a) front and (b) back violet irradiations (x means " not significant")

\section{Conclusions}

Tandem WDM a-SiC:H pi'n/pin devices are analyzed under different front and back optical bias wavelengths.

Results show that those devices, under appropriated optical bias act as reconfigurable active filters that allows optical switching and optoelectronic logic functions development. Depending on the wavelength of the external background the device acts either as a short- or a long- pass filter or as a band-stop filter.

An optoelectronic digital capture system based on this device is analyzed and the relationship between the optical inputs and the corresponding digital output levels established. A truth table of an encoder that performs 8-to-1 multiplexer (MUX) function is presented.

Acknowledgements. This work was supported by PTDC/EEA-ELC/111854/2009, PTDC/EEA-ELC/115577/2009 and PTDC/EEA-ELC/120539/2010. 


\section{References}

1. Connelly, M.J.: Semiconductor Optical Amplifiers. Springer, Boston (2002) ISBN: 978-07923-7657-6

2. Petit, C., Blaser, M.: Photodiodes with integrated optical filters for passive optical network applications. In: Fonjallaz, P.-Y., Pearsall, T.P. (eds.) Workshop on Optical Components for Broadband Communication. Proc. of SPIE, vol. 6350, p. 63500 (2006)

3. Ibrahim, S., Luo, L.W., Djordjevic, S.S., Poitras, C.B., Zhou, I., Fontaine, N.K., Guan, B., Ding, Z., Okamoto, K., Lipson, M., Yoo, S.J.B.: Fully reconfigurable silicon photonic lattice filters with four cascaded unit cells. In: Optical Fiber Communications Conference, OSA/OFC/NFOEC, San Diego (March 21, 2010); paper OWJ5

4. Yang, H., Jung, H.-D., Zheng, Y., Huiszoon, B., van Zantvoort, J.H.C., Tangdiongga, E., Koonen, A.M.J.: OFDM Radio-over-Fibre Systems Employing Routing in Multi-Mode Fibre In-Building Networks. In: Proc. ECOC 2008, Brussels, September 21-25 (2008); paper Tu.4.F.6

5. Yang, H., Lee, S.C.J., Tangdiongga, E., Okonkwo, C.M., Boom, H.P.A., van den Breyer, F., Randel, S., Koonen, A.M.J.: $47.4 \mathrm{~Gb} / \mathrm{s}$ transmission over $100 \mathrm{~m}$ graded-index plastic optical fiber based on rate-adaptive discrete multitone modulation. Journal of Lightwave Technology 28(4), 352-359 (2010)

6. Vieira, M., Louro, P., Fernandes, M., Vieira, M.A., Fantoni, A., Costa, J.: Three Transducers Embedded into One Single SiC Photodetector: LSP Direct Image Sensor, Optical Amplifier and Demux Device. In: Betta, G.F.D. (ed.) Advances in Photodiodes, ch. 19, pp. 403-425. InTech (2011) ISBN: 978-953-307-163-3

7. Louro, P., Vieira, M., Vieira, M.A., Fernandes, M., Costa, J.: Use of a SiC:H Photodiodes in Optical Communications Applications. In: Betta, G.F.D. (ed.) Advances in Photodiodes, ch. 19, pp. 377-402. InTech (2011) ISBN: 978-953-307-163-3 